\title{
Numerical Studies of Particle-Gas Two-Phase Flowing through Microshock Tubes
}

\author{
Guang Zhang, ${ }^{1}$ Wei Wei Wang, ${ }^{1}$ Xiang Hui Su, ${ }^{1}$ Xiao Jun Li $\mathbb{D}^{1},{ }^{1}$ Wen Hao Shen, ${ }^{2}$ \\ and Zhe Lin (D) 1 \\ ${ }^{1}$ State-Province Joint Engineering Lab of Fluid Transmission System Technology, Zhejiang Sci-Tech University, \\ Hangzhou 310018, China \\ ${ }^{2}$ Department of Oncology, Taizhou People's Hospital, Medical School of Nantong University, Taizhou, Jiangsu, China
}

Correspondence should be addressed to Zhe Lin; linzhe0122@zstu.edu.cn

Received 15 December 2020; Revised 24 January 2021; Accepted 3 February 2021; Published 15 February 2021

Academic Editor: Ling Zhou

Copyright ( 92021 Guang Zhang et al. This is an open access article distributed under the Creative Commons Attribution License, which permits unrestricted use, distribution, and reproduction in any medium, provided the original work is properly cited.

Microshock tubes are always used to induce shock waves and supersonic flows in aerospace and medical engineering fields. A needle-free drug delivery device including a microshock tube and an expanded nozzle is used for delivering solid drug powders through the skin surface without any injectors or pain. Therefore, to improve the performance of needle-free drug delivery devices, it is significantly important to investigate shock waves and particle-gas flows induced by microshock tubes. Even though shock waves and multiphase flows discharged from microshock tubes have been studied for several decades, the characteristics of unsteady particle-gas flows are not well known to date. In the present studies, three microshock tube models were used for numerical simulations. One microshock tube model with closed end was used to observe the reflected shock wave and flow characteristics behind it. The other two models are designed with a supersonic nozzle and a sonic nozzle at the exit of the driven section, respectively, to investigate particle-gas flows induced by different nozzles. Discrete phase method (DPM) was used to simulate unsteady particle-gas flows and the discrete random walk model was chosen to record the unsteady particle tracking. Numerical results were obtained for comparison with those from experimental pressure measurement and particle visualization. Shock wave propagation was observed to agree well with experimental results from numerical simulations. Particles were accelerated at the exit of microshock tube due to the reservoir pressure induced by reflected shock wave. Both sonic and supersonic nozzles were underexpanded at the end of microshock tubes. Particle velocity was calculated to be smaller than gas velocity, which results from larger drag of injected particles.

\section{Introduction}

During the past several decades, microshock tubes as devices to induce shock waves and supersonic flows have been widely used in mechanical, aerospace, and medical engineering fields, such as microcombustions, explosion, and needle-free drug delivery devices. Normally, a microshock tube consists of a driver section in high pressure and a driven section in low pressure which are separated by a thin diaphragm. Due to the pressure difference between two sections, shock waves are induced when the diaphragm is ruptured instantaneously [1]. If the diaphragm pressure ratio is extremely high, the diaphragm is ruptured naturally.
Otherwise, it should be punctured by using a needle manually. The incident shock wave induced by a microshock tube is always a normal shock wave, while it becomes oblique shock wave after it is reflected by the end wall in the driven section in a closed-ended microshock tube. Supersonic flows are induced and accelerated behind the incident shock wave, which is always applied in experimental tests such as microcombustion and explosion. High pressure and temperature flows are generated downstream of the reflected shock wave.

With the development of microshock tubes and the innovation of medical technique, needle-free drug delivery devices are designed to inject drug powders into human 
body in a contactless way. The main components of the needle-free drug delivery device are a microshock tube and an expanded nozzle [2]. Gas flows with drug powders are induced and accelerated by a shock wave generated in a microshock tube and accelerated in the expanded nozzle again. Drug powders are injected into the skin tissue by obtaining enough momentum. In order to ensure the delivery without any hurts, the momentum of drug powders should be strictly controlled. Even though needle-free drug delivery devices have been studied for several decades, detailed characteristics of particle-gas two-phase flows inside the devices are not well known to date. Experimental studies are sparsely conducted on particle velocity and momentum as well. However, investigations on particle-gas flows in needle-free drug delivery devices are extremely important in improving their performance in medical engineering fields.

Flows with solid particles behave differently from single gas flows. Due to the inertia and resistance of solid particles, particles are always not able to track gas flows properly. Under the slow relaxation time of solid particles, particles follow supersonic gas flows more improperly. The above effects make the momentum of solid particles uncontrollable, which prevents the development of needle-free drug delivery devices. Due to viscous effects and the existence of boundary layers, particle dynamics are more difficultly predicted in microshock tubes. Even though researchers paid great attention to investigating shock waves and particle-gas flows in microshock tubes, detailed characteristics of shock wave and particle dynamics were not well known.

Brouillette [1] derived a new model to investigate scale effects on shock wave generation and propagation in a microshock tube and performed a comparison with experimental results. A control volume located between shock wave and contact surface was considered. Particle velocity was lower than that in microshock tubes in larger scales. The predicted numerical results agreed with experimental results well with respect to the Mach numbers of shock waves. Liu et al. [2] performed experimental and numerical investigations on shock wave propagation and particle velocity in a contoured shock tube. The momentum of drug powders was controlled by flow characteristics inside the contoured shock tube. Austin et al. [3] studied shock wave propagation and attenuation through a microscale channel of circular cross section. Shock wave velocity and pressure histories from experimental studies were compared with experimental and theoretical results. More shock wave attenuation was observed in the channels with smaller scales and lower pressures. Felling et al. [4] used pressure measurements to record pressure histories compared with existing experimental pressure results.

Effects of the pressure ratio and diaphragm location were investigated on shock waves discharged from an open-ended shock tube. Mach-disk shock, barrel shock, and reflected shock waves were observed and discussed in detail by Haselbacher et al. [5]. Labastida et al. [6] used simultaneous lateral and end-wall high-speed visualization method to observe shock wave propagation in a circular shock tube. Shock wave structure and motion were obviously visualized. A modified dissipation model was derived to discuss effects of heat transfer on shock velocity gradient by Yang et al. [7]. By considering the effects of Reynolds number and temperature difference between particle and gas phases, Henderson [8] derived mathematic models to calculate particle drag force under subsonic and supersonics gas-particle flows, respectively. Lin et al. [9-13] used CFD-EDM coupled method to calculate particle-gas flows and carried out visualization tests to validate CFD results. Particle trajectory and velocity from numerical simulations agreed with experimental results well. Sun et al. [14-19] made summaries on numerical models of calculating multiphase flows and derived some new mathematic models to calculate multiphase flows.

Numerical simulations were carried out to investigate propagation and attenuation of shock wave in micro shock tubes by using a one-dimensional approach by Ngomo et al. [20]. Attenuation of shock wave was quantitatively calculated and propagation of shock wave was obviously captured at different tube diameters. Li et al. [21] conducted timeresolved shadowgraph and transient pressure measurements to investigate shock wave structure and Mach number in a shock tube. Shock flows were discussed at the tube exit in detail. Digital particle image velocimetry was used to capture shock wave structure at the nozzle exit impinging to a plate by Henderson et al. [22]. Nozzle pressure ratio and exit diameter were investigated to have great effects on shock flows at the nozzle exit. Kendall et al. [23-25] performed experimental studies to investigate particle dynamic through contoured shock tubes by particle image velocimetry (PIV) and Schlieren visualization. The velocity and propagation of shock wave were obtained and discussed in detail.

Interaction between shock wave and solid particle was investigated by Xiong et al. [26]. Shock wave was observed to affect particle cloud clusters and small particle influenced shock wave structure as well. In addition, a controlling method for cloud cluster expansion rate was proposed. Lupoi et al. [27] conducted numerical and experimental studies to investigate particle behavior in a supersonic nozzle for cold spray system. The collisional model was derived to calculate particle dispersion in supersonic flows, which agreed with experimental results well. Wang et al. [28] derived a unified gas-kinetic scheme to calculate particle-gas flows. Collision of two particle-gas phases was discussed in detail as well as shock-driven multiphase instability. Experimental investigations were carried out to study shock wave dynamic and particle movement in microshock tubes from previous studies $[29,30]$. Shock wave and particle motion were visualized and analyzed in detail.

In this article, numerical studies on shock wave propagation and particle motion were carried out in different microshock tubes. By considering solid particles behaving differently in supersonic and subsonic flows, a suitable drag coefficient model was used in present numerical simulations. Shock flows and particle motion induced by sonic and supersonic nozzles at the tube exit were obtained and discussed in detail, respectively. The comparisons were made between CFD and experimental results on pressure histories inside the microshock tube and at different nozzle exits. Shock wave propagation was visualized and compared with 
results got from particle tracking velocimetry. Particle velocities induced by sonic and supersonic nozzles were obtained and discussed in detail.

\section{Numerical Methods}

2.1. Computational Domain. A microshock tube was designed to investigate characteristics of unsteady particlegas flows as shown in Figure 1. The shock tube was designed with the driver section in a diameter of $20 \mathrm{~mm}$ and a length of $41 \mathrm{~mm}$, and the diameter and length of the driven section are $7.5 \mathrm{~mm}$ and $66 \mathrm{~mm}$, respectively. All sections have circular cross sections. Sonic and supersonic nozzles were, respectively, installed at the end of the driven section in the microshock tube. The detailed schematic and size of the sonic and supersonic nozzles are shown in Figures 2(a) and 2(b). Pressure measurement and Schlieren visualization were conducted.

Numerical simulations were carried out by using the same model as the experimental model. Due to circular shape of microshock tube, $2 \mathrm{D}$ axisymmetric domain was used as shown in Figure 3. Two points located at the tube wall of driven section working as the locations of pressure transducers in experimental tests. The static pressure histories were recorded at two points, so the shock wave strength as well as velocity was obtained. In order to investigate the particle acceleration, sonic and supersonic nozzles were installed at the end of the driven section, which acts as a contoured shock tube for a needle-free drug delivery device. The detailed schematic of sonic and supersonic nozzles is shown in Figure 2.

2.2. Governing Equations. Supersonic flows can be predicted by using mass, momentum, and energy equations:

$$
\begin{gathered}
\frac{\partial \rho_{v}}{\partial t}+\frac{\partial}{\partial x_{i}}\left(\rho_{v} u_{j}\right)=0 \\
\frac{\partial}{\partial t}\left(\rho_{v} u_{j}\right)+\frac{\partial}{\partial x_{j}}\left(\rho_{v} u_{j} u_{j}\right) \\
=\frac{\partial p_{v}}{\partial x_{i}}+\frac{\partial}{\partial x_{j}}\left[\eta\left(\frac{\partial u_{j}}{\partial x_{i}}+\frac{\partial u_{i}}{\partial x_{j}}+\frac{2}{3} \delta_{i j} \frac{\partial u_{j}}{\partial x_{j}}\right)\right]+\frac{\partial}{\partial x_{j}}\left(-\rho_{v} u_{i}^{\prime} u_{j}^{\prime}\right), \\
\frac{\partial}{\partial t}\left(\rho_{v} E\right)+\frac{\partial}{\partial x_{j}}\left(\rho_{v} u_{j} E+u_{j} p_{v}\right)=\frac{\partial}{\partial x_{j}}\left(k_{\mathrm{eff}} \frac{\partial T}{\partial x_{j}}+u_{i} \tau_{\mathrm{eff}}\right),
\end{gathered}
$$

where $u, \rho, p, \mu$, and $T$ represent flow velocity, density, pressure, viscosity, and temperature, respectively. $\delta$ is Kronecker delta and $E$ is total energy. $k_{\text {eff }}$ and $\tau_{\text {eff }}$ are effective thermal conductivity and effective stress tensor, respectively.

Turbulence model is significantly important for calculating supersonic flows. In present studies, $k-\omega$ turbulence model was used due to its good applicability for calculating

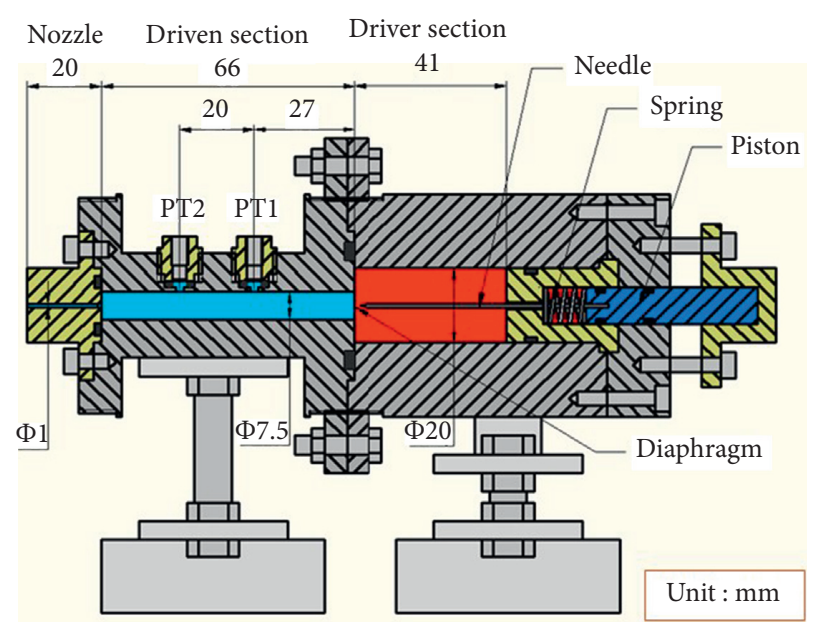

Figure 1: Experimental microshock tube.

compressible flows and wall bounded flows. Turbulent kinetic energy $k$ and dissipation rate $\omega$ are calculated by

$$
\begin{gathered}
\frac{\partial}{\partial t}\left(\rho_{v} k\right)+\frac{\partial}{\partial x_{j}}\left(\rho_{v} k u_{i}\right)=\frac{\partial}{\partial x_{j}}\left(\Gamma_{k} \frac{\partial k}{\partial x_{j}}\right)+G_{k}-Y_{k}, \\
\frac{\partial}{\partial t}\left(\rho_{v} k\right)+\frac{\partial}{\partial x_{j}}\left(\rho_{v} \omega u_{i}\right)=\frac{\partial}{\partial x_{j}}\left(\Gamma_{\omega} \frac{\partial \omega}{\partial x_{j}}\right)+G_{\omega}-Y_{\omega},
\end{gathered}
$$

where $G_{k}$ and $G_{\omega}$ are turbulent kinetic energy and dissipation rate at average velocity gradients, respectively. $Y_{k}$ and $Y_{\omega}$ represent the dissipation of $k$ and $\omega$ at the turbulent state. $\Gamma_{k}$ is effective dissipation rate of turbulent kinetic energy and $\Gamma_{\omega}$ is effective dissipation rate of the dissipation rate.

2.3. Particle Drag Coefficient. Solid particles have different force characteristics under effects of supersonic and sonic flows [8]. By assuming that particles are spherical, drag coefficients of solid particle are calculated in subsonic and supersonic flows based on equations (6) and (7), respectively.

For subsonic flow,

$$
\begin{aligned}
C_{D}= & 24\left\{\operatorname{Re}+S\left[4.33+1.567 * \exp \left(-0.247 \frac{\mathrm{Re}}{S}\right)\right]\right\}^{-1} \\
& +\exp \left(-0.5 \frac{M}{\sqrt{\mathrm{Re}}}\right)\left[\frac{4.5+0.38(0.03 \mathrm{Re}+0.48 \sqrt{\mathrm{Re}})}{1+0.03 \mathrm{Re}+0.48 \sqrt{\mathrm{Re}}}\right. \\
& \left.+0.1 M^{2}+0.2 M^{8}\right]+\left[1-\exp \left(-\frac{M}{\mathrm{Re}}\right)\right] * 0.6 S .
\end{aligned}
$$

For supersonic flow $\left(M_{\mathrm{g}}>1.75\right)$,

$$
C_{D}=\frac{0.9+0.34 / M^{2}+1.86(M / \mathrm{Re})^{1 / 2}\left[2+2 / S^{2}+1.058 / S+1 / S^{4}\right]}{1+1.86(M / \mathrm{Re})^{1 / 2}} .
$$

As particles moves in supersonic flows at Mach number between 1 and 1.75, a modification of equation (7) is given as 


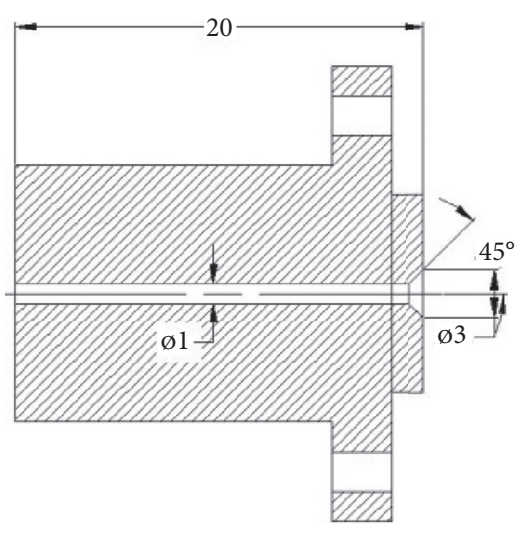

(a)

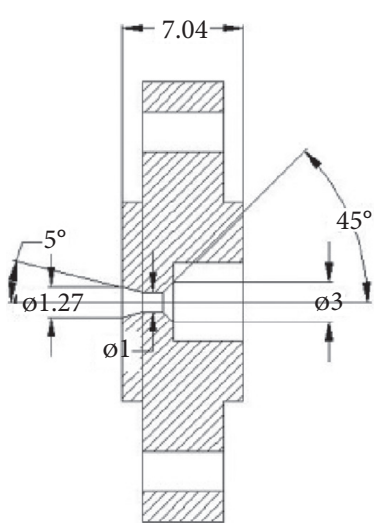

(b)

FIgURE 2: Sonic and supersonic nozzle models installed at the exit of the driven section. (a) Sonic nozzle. (b) Supersonic nozzle.

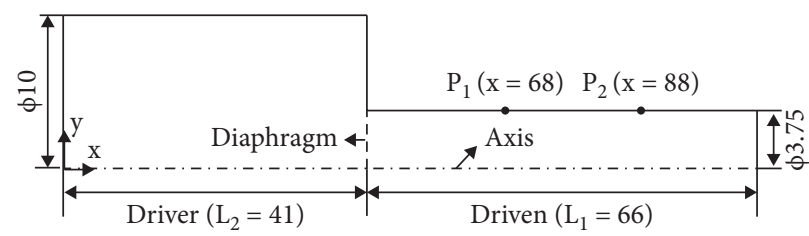

Figure 3: Computational domain for numerical simulations.

$$
C_{D}=\frac{24}{\operatorname{Re}} \frac{1+\exp \left(-\left(0.427 / M^{4.63}\right)-\left(3 / \mathrm{Re}^{0.88}\right)\right)}{1+M / \operatorname{Re}[3.82+1.28 \exp (-1.25 \operatorname{Re} / M)]}
$$

where $C_{\mathrm{D}}$ is drag coefficient and Re is particle Reynolds number. $M_{\mathrm{g}}$ is Mach number of gas phase. $M$ is Mach number based on relative velocity between gas phase and particle phase, and $S$ is the molecular speed ratio. Particle Reynolds number is calculated by the following equation:

$$
\operatorname{Re}=\frac{\rho_{P}\left|U-U_{P}\right| D_{P}}{\mu}
$$

where $\rho_{P}$ is particle density and $D_{P}$ is particle diameter. $\mu$ is dynamic viscosity of fluid and $U$ and $U_{P}$ are the velocities of gas phase and particle phase, respectively. Particle drag forces $F_{D}$ can be obtained based on above expressions of particle drag coefficients at different flow conditions as shown in Equation 10. UDF were written on particle drag coefficient and drag force and inserted in Ansys Fluent to calculate particle motion in supersonic and subsonic flows.

$$
F_{D}=\frac{3 \mu C_{D} \operatorname{Re}}{4 \rho_{P} D_{P}^{2}} .
$$

The correlation on drag coefficients was incorporated in Ansys Fluent by user defined function (UDF) for numerical simulations on particle dynamics.

2.4. Boundary Conditions. Ansys Fluent was used to calculated two-phase flow of particle and gas in microshock tubes. k- $\omega$ shear stress transport (SST) was chosen as turbulence model due to its good capacity in calculating unsteady flows with high pressure gradient and boundary layers near walls. The minimum grid size determined the time step of unsteady calculations to be $10^{-7} \mathrm{~s}$. All walls were assumed to be adiabatic and kept at constant temperature of $300 \mathrm{~K}$, so no heat transfer happened between the shock heated flows and tube walls in numerical simulations. Working fluid was assumed as ideal gas in the driver and driven sections. Particle motion was calculated based on discrete phase model (DPM) regarding gas flow as continuous phase and particle as discrete phase for particle-gas flows. Two-way turbulence coupling model was used to consider the interaction between particle phase and gas phase. Discrete random walk model and unsteady particle tracking method were chosen to track unsteady particle behavior. Assuming the particle as the sphere, drag law proposed by Henderson [8] was used to calculate particles dispersion in supersonic flows. Anthracite is chosen as seeding particle in the present CFD studies, which has the same $10 \mu \mathrm{m}$ as previous experimental studies and the density of $1020 \mathrm{~kg} / \mathrm{m}^{3}$. Particles were injected at the position of the diaphragm with particle mass flows rate of $0.001 \mathrm{~kg} / \mathrm{s}$ and seeding velocity of $0.5 \mathrm{~m} / \mathrm{s}$.

2.5. Mesh Independence Study. Structured quad grids were created for full computation domain as shown in Figure 4(a). To indicate the interaction between shock wave and boundary layer, boundary layer grids were drawn near tube walls. In order to capture the detailed structure of moving shock waves and particle motion, creating high-quality grids at the direction of moving shock wave is significantly important. A suitable number of grids are used to capture shock wave propagation and save time of calculations. Three 


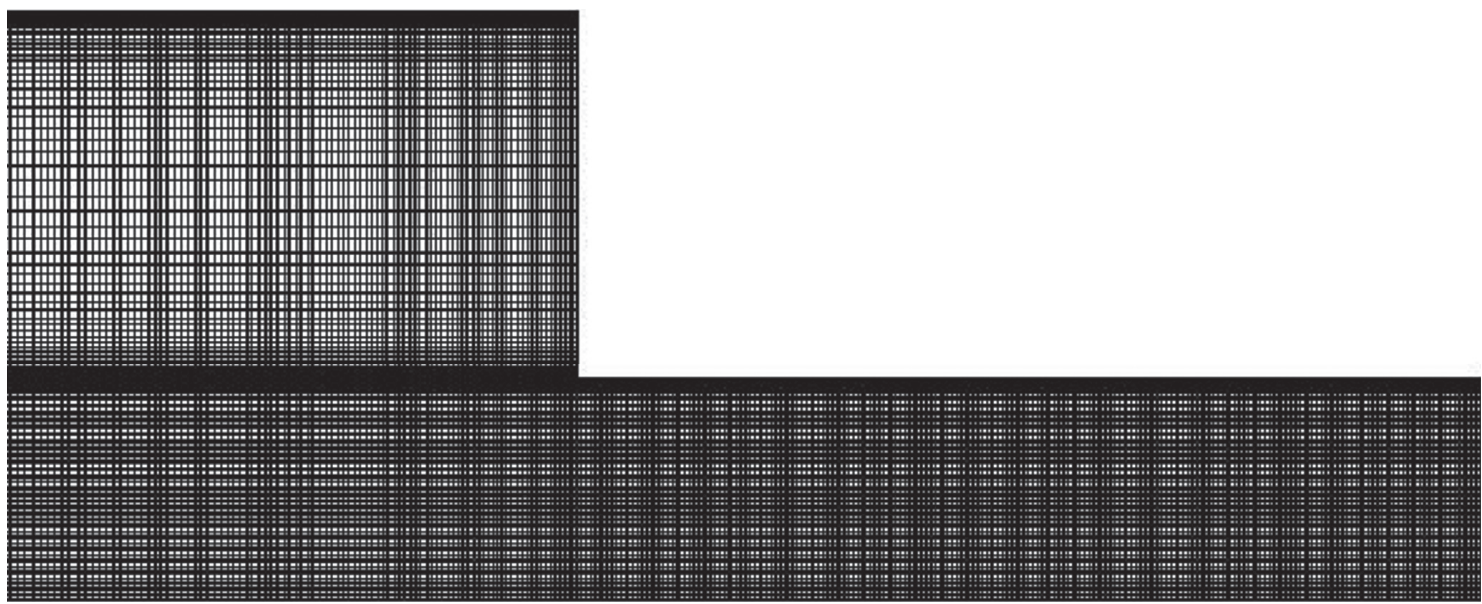

(a)

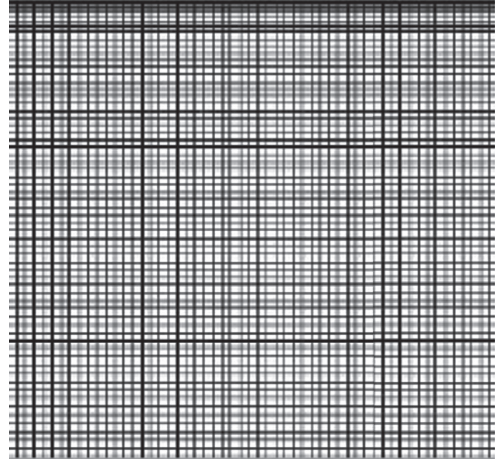

(b)

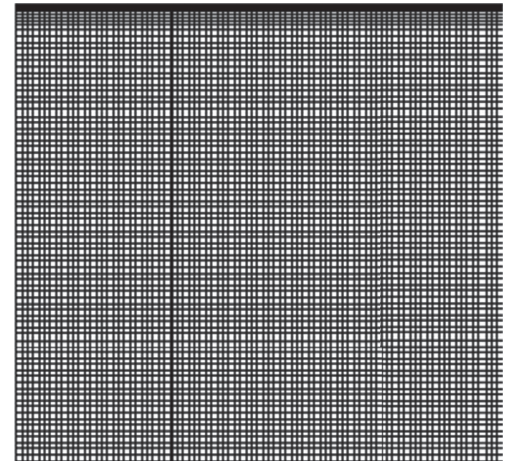

(c)

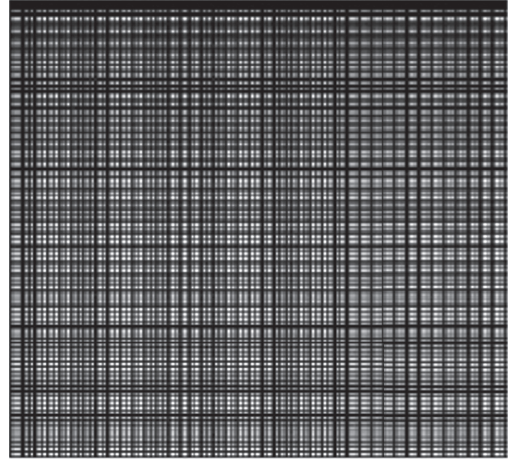

(d)

Figure 4: Partial computational grids used for numerical simulation. (a) Partial computational grids. (b) 89000. (c) 192000. (d) 256000.

kinds of grids were used to validate mesh independence studies. The mesh numbers for three cases are 89000, 192000, and 256000, respectively, as shown in Figures 4(b)4(d). Pressure histories of point 1 are shown in Figure 5. Before the normal shock wave was reflected, pressure histories were almost the same among three cases. A slight difference was observed on pressure histories after the shock wave met the end wall of microshock tube. The deviation happened due to the less number of meshes used in the case of the mesh number of 89000 . In addition, pressure histories obtained from the grid size of 192000 were observed to be almost the same as those obtained from the grid size of 256000 and two curves of pressure histories were almost superposed. Based on the accuracy and time of calculations, the mesh size of 192000 was chosen and used for the present simulations.

\section{Results and Discussion}

3.1. Pressure Histories in the Driven Section. All experimental tests and numerical simulations were performed at the same conditions. The driven section was kept in constant pressure of the atmosphere pressure and the driver section was initialized in high pressure of $0.9 \mathrm{MPa}$, which fixed the diaphragm pressure ratio to be 9 for all cases. The comparison between the experimental and numerical pressure histories is shown in Figure 6.

After the diaphragm was ruptured, the incident normal shock wave was induced and moved towards the driven section. As the shock wave reached the position where pressure transducers located, the pressure increased steeply as shown in Figure 6. The instants where the shock wave met the two pressure transducers were almost similar in both experimental and numerical studies. The strength of the shock wave was observed to be stronger in CFD study compared to that in experimental test, which mainly resulted from the difference in rupture process. In experimental tests, the diaphragm was ruptured by the manual method and the rupture time was not instantaneous. However, the instantaneous rupture was simulated as the boundary condition of the diaphragm was changed from the wall to the interior. In addition, the $2 \mathrm{D}$ half computational domain was used in CFD study, but actually the shock wave moving in the microshock tube was not symmetrical with respect to the center line in the experimental test. The adiabatic walls were used for the numerical simulations, so the heat transfer between the shock heated air and tube walls was ignored. However, heat transfer existed in the experimental study. 


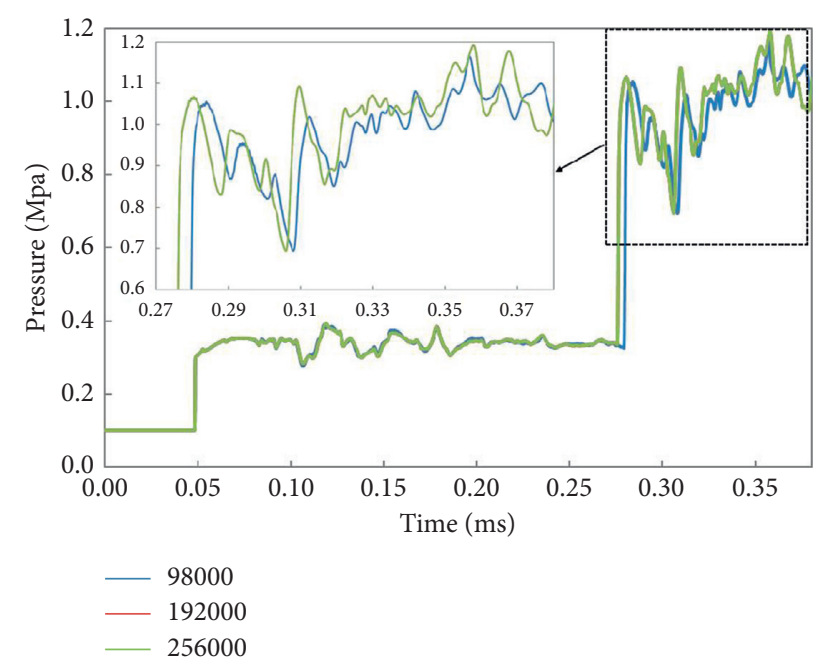

FIgURE 5: Pressure histories of point 1 for different computational grids.

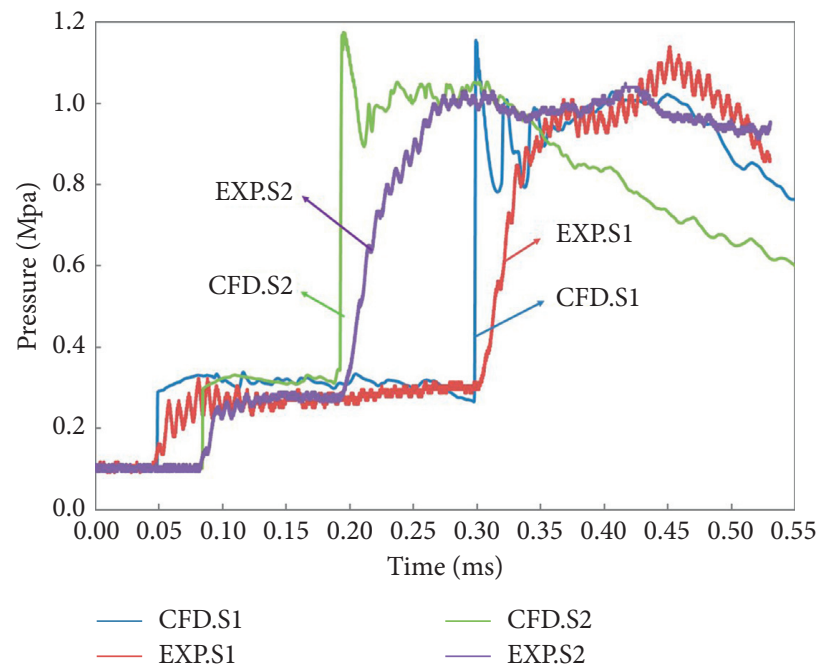

FIgURE 6: Pressure histories for the case with closed end in the driven section.

As the shock wave met the end wall of the driven section, it was reflected and moved towards the direction opposite to the incident shock wave. The deviation between experimental and CFD results gradually became larger. This was mainly due to the fact that the shock wave experienced much more decay after it was reflected in the experimental test. As the shock wave collided to the solid wall, much more shock wave attenuation occurred.

3.2. Shock Wave Propagation. In previous experimental studies, Schlieren visualization was used to observe the shock wave propagation in microshock tube as shown in Figure 7 . The test section was clearly observed before the normal shock wave was induced. After the diaphragm was ruptured, a normal shock wave was induced and propagated to the end wall as shown in Figures 7(b) and 7(c). When the normal shock wave met the end wall of the driven section, it was reflected and the reflected shock wave was still normal shock wave as shown in Figure $7(\mathrm{~d})$. The strength of the primary shock wave was stronger than that of the reflected shock wave. This resulted from the fact that the reflected shock wave moved upstream. As reflected shock wave met the contact surface, the structure of the reflected shock wave changed as shown in Figure 7(e). Reflected shock wave was no longer a normal shock wave but the direction did not change. The dark spots and areas shown in the figures were particles seeded in the microshock tube.

Numerical simulations were carried out to investigate the shock wave propagation and compared with experimental results. Temperature contours at different time are shown in Figure 8. The shock wave and the contact surface are clearly observed. Shock wave propagations from experimental and CFD studies agreed well and shock wave structures were also similar. After the shock wave was reflected, a high-temperature region occurred behind the reflected shock wave. This showed a good agreement with the previous observation that the high pressure developing 


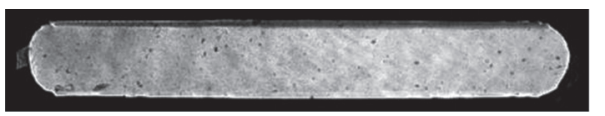

(a)

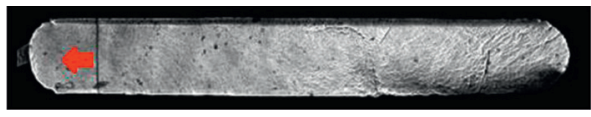

(c)

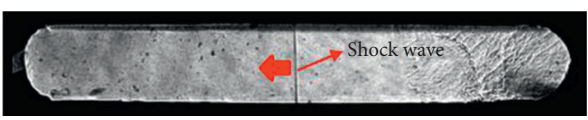

(b)

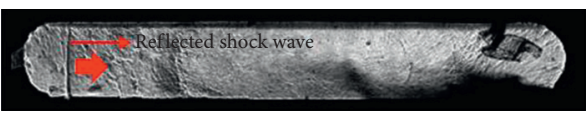

(d)

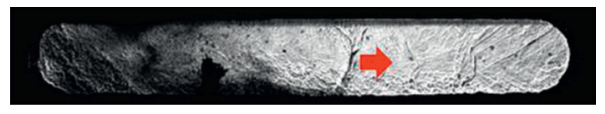

(e)

FIGURE 7: Shock wave propagation from experimental visualization.

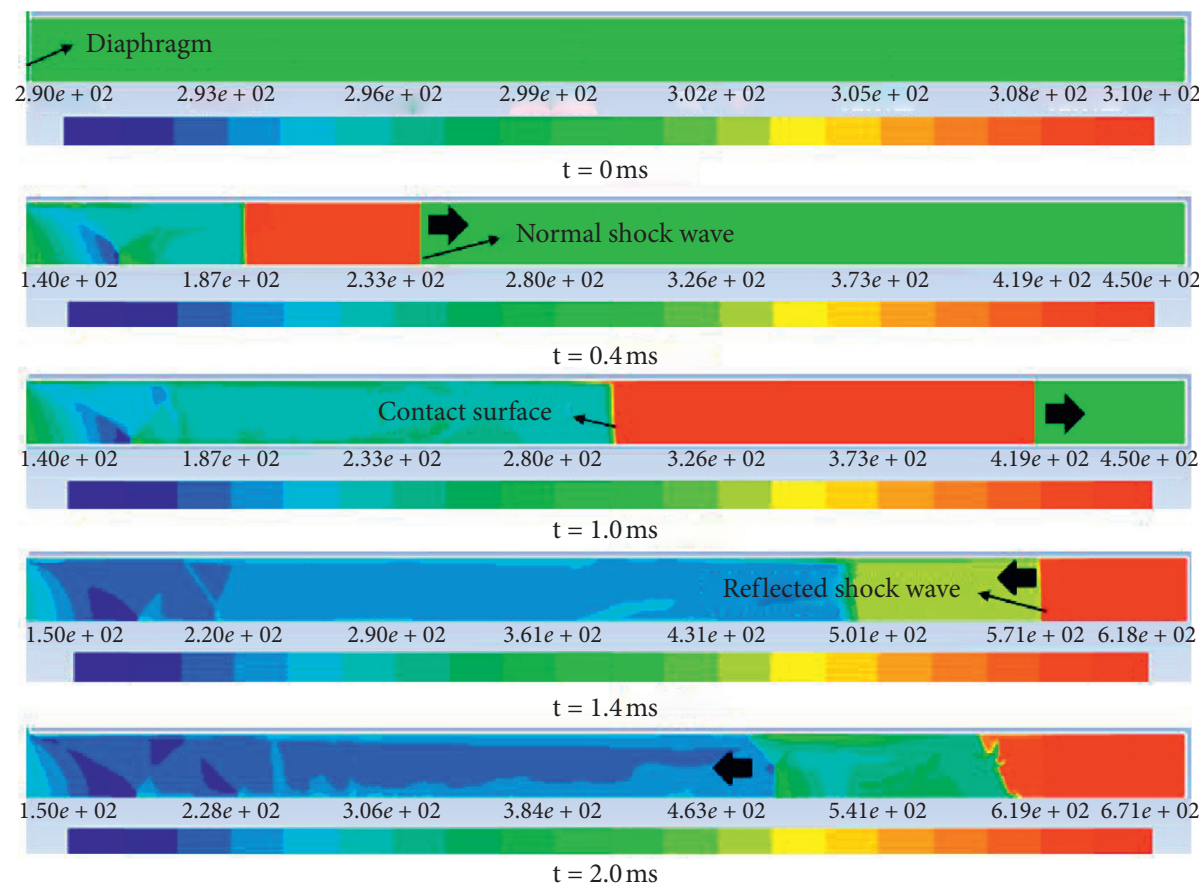

FIgURE 8: Temperature contours inside the driven section from numerical simulations.

behind the reflected shock wave was regarded as reservoir pressure for initializing nozzle flows. As the reflected shock wave met the contact surface, its structure changed and the shock wave was not normal any more.

\subsection{Pressure Histories Downstream of Nozzle Exit. After the} normal shock wave was reflected by the end wall, high pressure and temperature flows were generated behind the reflected shock wave. The high pressure flows acted as the reservoir pressure to induce nozzle flows. Pressure histories were recorded downstream of the nozzle exit and compared as shown in Figure 9. The bow shock wave in front of Pitot tube was observed as the discontinuous pressure changes as shown in both experimental and numerical studies. Experimental pressure histories measured by the Pitot tube agreed with the numerical results well except that the instant at which the supersonic flows moved through the measured position was different. The supersonic flows reached the measured position earlier in numerical simulation compared to those in experimental test. This is due to the earlier reflection of the shock wave in the CFD study, which was caused by less shock wave attenuation happening in the CFD study compared to that in experimental test. The pressure histories at the exit of the supersonic nozzle are shown in Figure 9 as well and the trend of pressure changes was similar to that in the case of the sonic nozzle. The reservoir pressure values were lower at the exit of the sonic nozzle $(0.46 \mathrm{MPa})$ compared to those at the exit of the supersonic nozzle $(0.52 \mathrm{MPa})$. In addition, the time when shock flows moved through the monitor point at the exit of supersonic nozzle was earlier than that at the exit of sonic nozzle. This is 


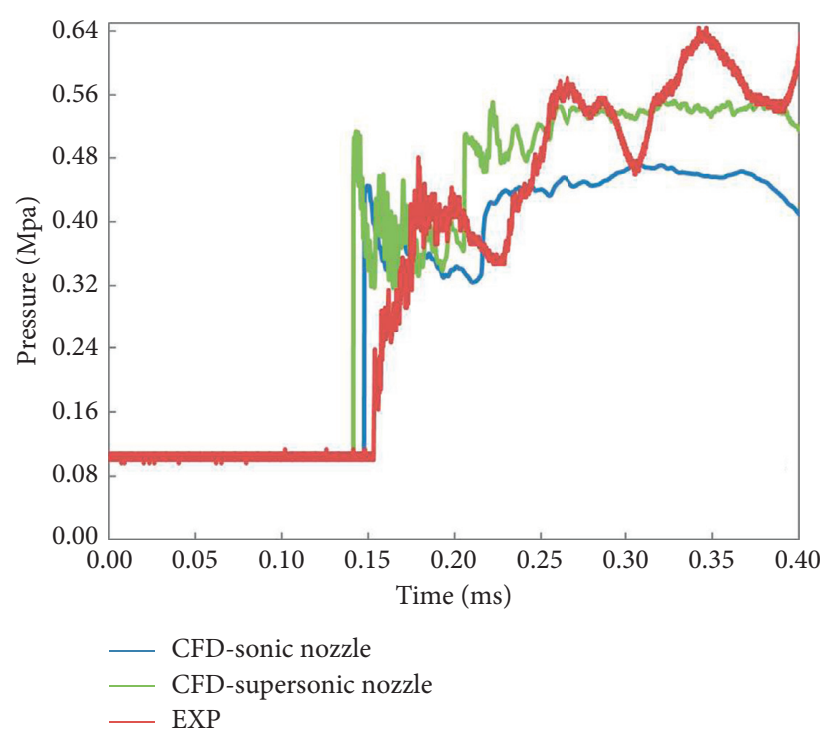

FIgURE 9: Pressure histories at the exit of sonic and supersonic nozzles.

due to different positions where monitor points located as shown in Figure 2. The monitor point was installed downstream of the exit of the sonic nozzle, where a long constant channel was designed to support the installation of pressure transducer in experimental tests. However, no constant channel was designed at the exit of supersonic nozzle and the monitor was set at the center of the exit of supersonic nozzle in CFD studies, which is much closer to the end of the driven section of microshock tube.

3.4. Particle Velocity. Particle motion was clearly observed in the driven section as shown in Figure 10. As solid particles moved inside the microshock tube, the particle velocity gradually increased. Boundary layer developed behind the moving shock wave, which made the motion of particle-gas flows similar to particle-gas flows moving through a nozzle; therefore, the particle velocity increased. Particle velocity decreased as the reflected shock wave came across particles. When the incident shock wave met the end wall of the driven section, it was reflected and moved upstream. At $t=0.5 \mathrm{~ms}$, some particles moved to the driver section opposite to the previous direction and particles highly attenuated in the front of the particle distribution. Particles inside the boundary had a low velocity. When meeting the reflected shock wave, particles velocity was decreased and moved towards the driver section by following the reflected shock wave. Particles in front of the particle distribution map moved into high pressure region, so particle velocity was decreased. The particle velocity was calculated as shown in Figure 11. Particle velocities induced by sonic and supersonic nozzles were observed to be almost the same before particles met the reflected shock wave. There was a slight difference after they came cross the reflected shock wave. Particles inside the driven section were not accelerated to supersonic velocity in the present CFD simulations.

Particle motion and velocity contours of gas flows induced by the sonic nozzle are, respectively, shown in Figures 12(a) and 12(b). Due to the underexpanded nozzle flows, particle velocity gradually increased downstream of the sonic nozzle exit. The expansion waves were clearly observed at the exit as shown in Figure 12(b). Compared to the velocity of gas flows, the particle velocity was much lower, which resulted from the large diameter of particles. Large particles had large inertia and resistance, making particles follow gas flows improperly. As the reservoir pressure decreased in the driven section, the expansion waves became weak and particle velocity also decreased. The similar characteristics of particles and gas flows generated by the supersonic nozzle were observed compared to those induced by the sonic nozzle as shown in Figures 13(a) and 13(b). Higher particle velocity was observed at the exit of sonic nozzle compared to that at the exit of supersonic nozzle. This resulted from the fact that the flows generated by the sonic nozzle were more highly underexpanded. This is clearly shown as the flow velocity induced by the sonic nozzle was much higher at the nozzle exit.

In both CFD and experimental studies, particle velocity was obtained in four test sections downstream of nozzle exit as shown in Figure 14. The test section was defined in area of $1 \mathrm{~mm} \times 3.36 \mathrm{~mm}$ with the interval of $1 \mathrm{~mm}$ from $X=0 \mathrm{~mm}$ to 


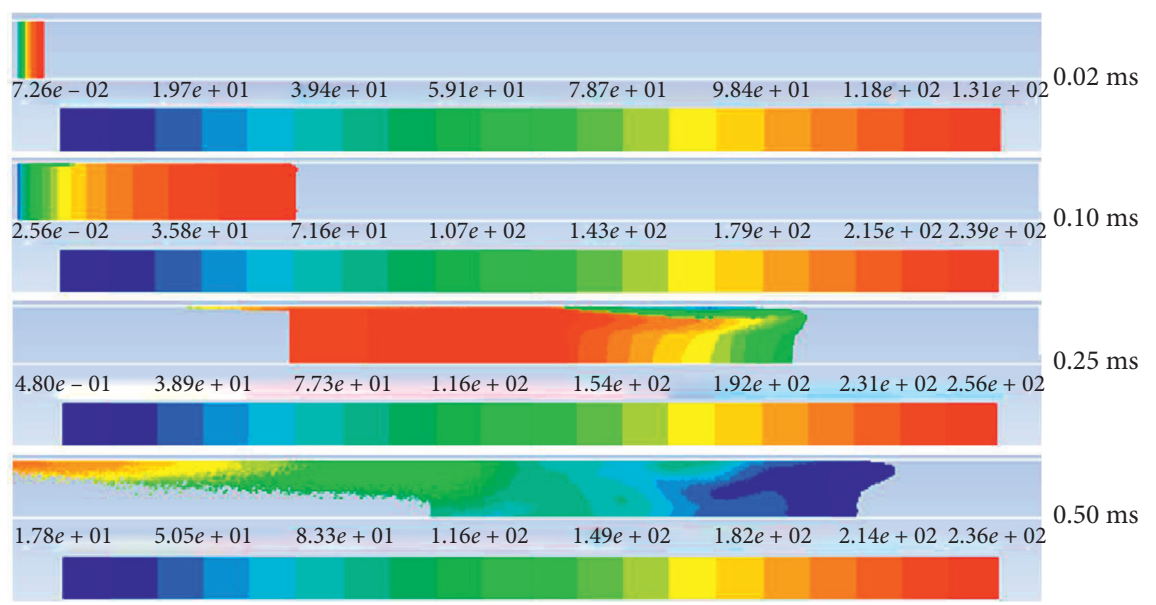

FiguRE 10: Particle velocity distributions in the driven section.

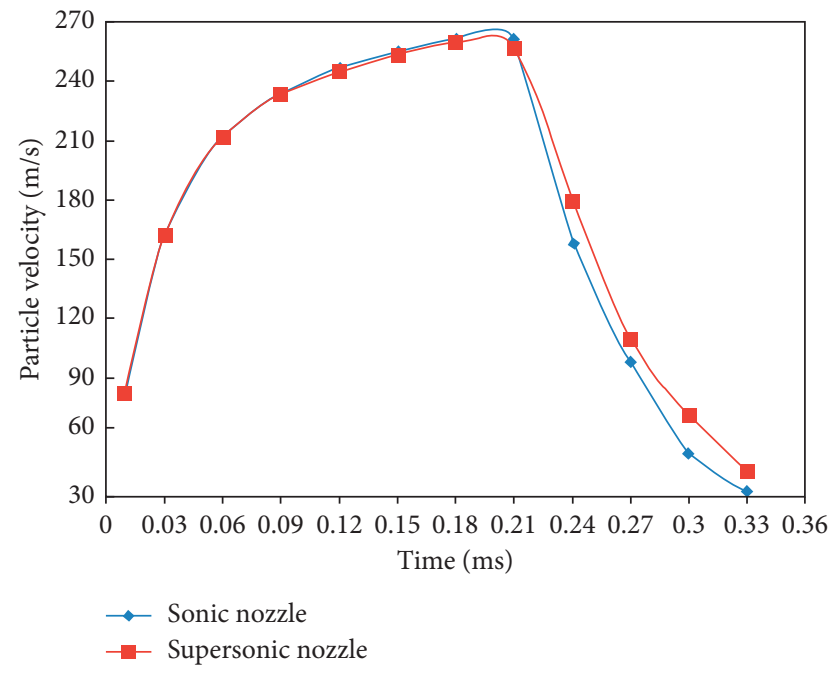

FIgure 11: Particle velocity inside the driven section.
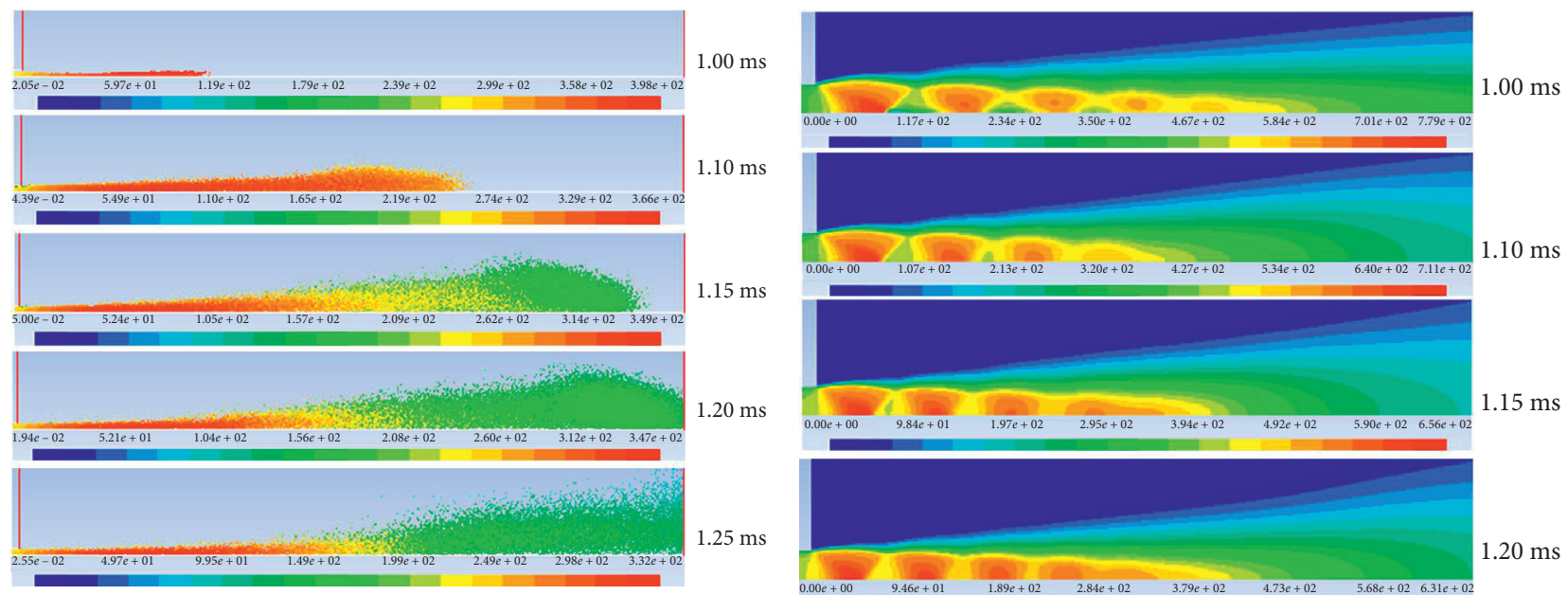

$1.15 \mathrm{~ms}$

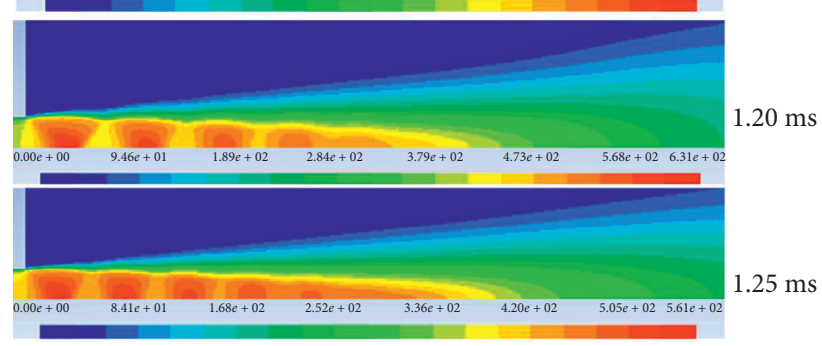

$1.20 \mathrm{~ms}$

(a)

(b)

FIgURe 12: Particle distributions and Mach number contours at the exit of sonic nozzle. (a) Particle distributions. (b) Mach number contours. 


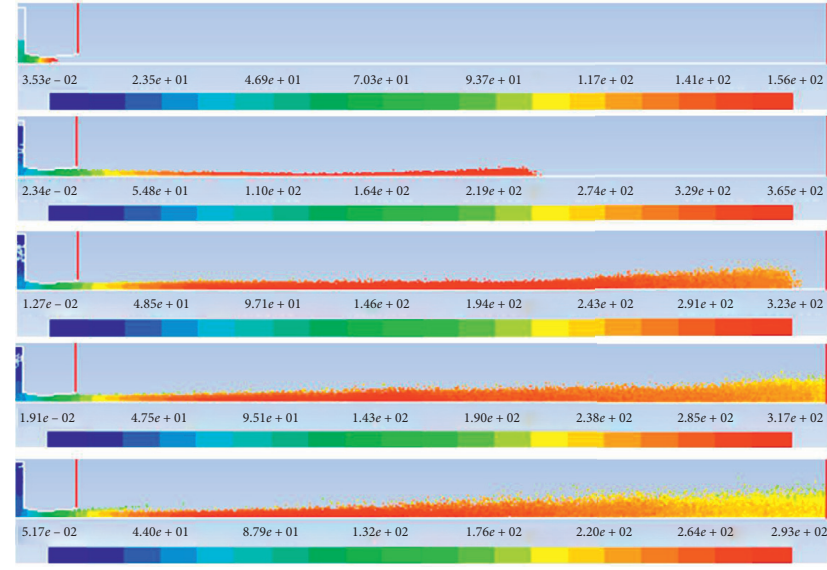

(a)

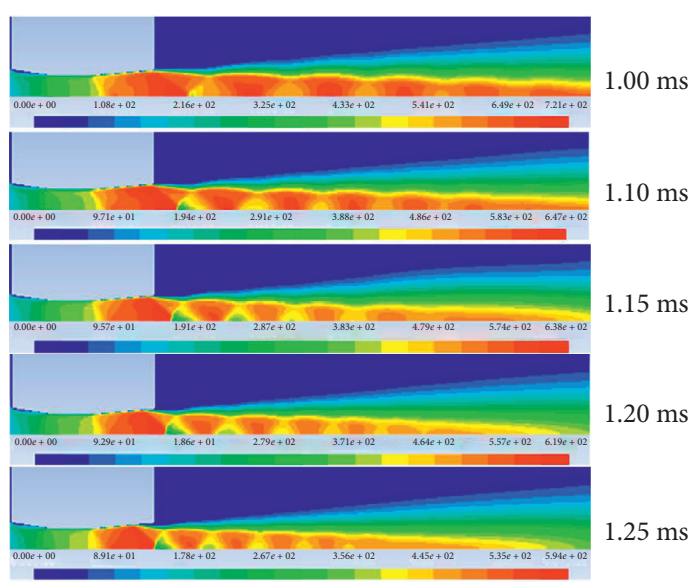

(b)

Figure 13: Particle distributions and Mach number contours at the exit of supersonic nozzle. (a) Particle distributions. (b) Mach number contours.

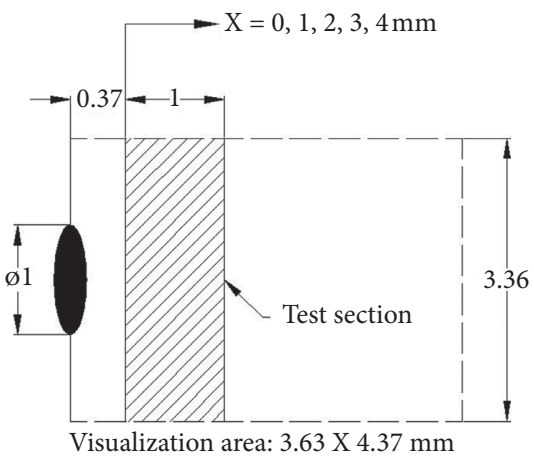

FIGURE 14: Test sections for visualization.

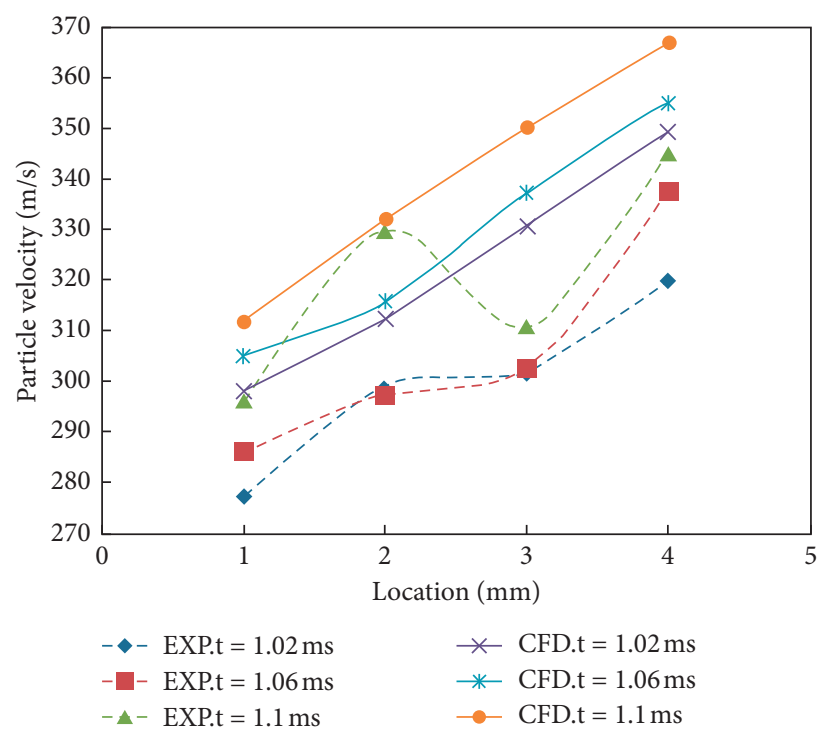

FIgURE 15: Particle velocity at the exit of sonic nozzle. 
$X=4 \mathrm{~mm}$. Similar test sections were used in the CFD simulations. Particle velocity was calculated for the numerical simulation and compared to the results obtained in the experimental studies as shown in Figure 15. As particles moved outside of the nozzle exit, particle velocity gradually increased in both CFD and experimental studies. This is due to the fact that the sonic nozzle flows were underexpanded at the nozzle exit. Particle velocity obtained from the CFD studies was higher compared to that in experimental calculations. The main reason is that the shock wave strength was observed to be stronger in the numerical simulation as mentioned previously, so the reservoir pressure generating in the driven section was also higher in the CFD study.

\section{Conclusions}

Numerical simulations were carried out to study shock wave propagation and particle-gas flows induced by sonic and supersonic nozzles and the comparison between numerical and experimental results was made as well. Normal shock wave and reflected shock wave were clearly observed in CFD studies and agreed with experimental results well. Pressure histories indicated that less shock wave attenuation happened in the CFD simulations compared to that in the experimental studies, which resulted from the different methods used for rupturing diaphragms. In CFD studies, the diaphragm was ruptured instantaneously instead of manual rupturing method by using a needle. In addition, much more viscous effects and friction between the shock wave front and tube walls took place in the experimental tests. After the normal shock wave met the end wall, high pressure and temperature flows were induced behind the reflected shock wave. This made the high pressure the reservoir pressure inducing nozzle flows. Particles were accelerated in the microshock tube due to the development of boundary layers behind the shock wave. Both sonic and supersonic nozzle flows were choked and shock wave structure was clearly observed. Particles were observed to be accelerated behind the exit of both sonic and supersonic nozzles due to both nozzle flows being underexpanded. Particle velocity showed large deviation from the velocity of gas flows, which resulted from large particle diameter used in the present studies. In the future studies, the small particle diameter will be considered and investigated in the microshock tube.

\section{Data Availability}

The data used to support the findings of this study are available from the corresponding author upon request.

\section{Conflicts of Interest}

The authors declare no conflicts of interest with respect to the research, authorship, and/or publication of this article.

\section{Acknowledgments}

This research was funded by the National Natural Science Foundation of China (Grants nos. 51906222 and 51706206), Key Research and Development Program of Zhejiang
Province (Grant no. 2019C03117), and Zhejiang Sci-Tech University (Grant no. 18022135-Y). The authors acknowledge the financial support.

\section{References}

[1] M. Brouillette, "Shock waves at microscales," Shock Waves, vol. 13, no. 1, pp. 3-12, 2003.

[2] Y. Liu and M. A. F. Kendall, "Numerical analysis of gas and micro-particle interactions in a hand-held shock-tube device," Biomedical Microdevices, vol. 8, no. 4, pp. 341-351, 2006.

[3] J.-M. Austin and D.-J. Bodony, "Wave propagation in gaseous small-scale channel flows," Shock Waves, vol. 21, no. 6, pp. 547-557, 2001.

[4] M.-M. Felling, R.-H. Page, H.-H. Korst, and R.-A. White, “An experimental analysis and demonstration of the non-steady flow in a shock tube," International Journal of Engineering Education, vol. 14, no. 1, pp. 59-66, 1998.

[5] A.-C. Haselbacher, S. Balachandar, and S.-W. Kieffer, "Openended shock tube flows: influence of pressure ratio and diaphragm position," AIAA Journal, vol. 45, no. 8, p. 1917, 1929.

[6] M.-F. Labastida and A. Farooq, "Simultaneous lateral and endwall high-speed visualization of ignition in a circular shock tube," Combustion and Flame, vol. 214, pp. 263-265, 2020.

[7] Y. Yang, I. Kim, and G. Park, "Evaluation of blunt body velocity gradient at the shock tube end-wall," Acta Astronautica, vol. 170, pp. 570-576, 2020.

[8] C.-B. Henderson, "Drag coefficients of spheres in continuum and rarefied flows," AIAA Journal, vol. 14, no. 6, pp. 707-708, 1976.

[9] Z. Lin, Z. Liu, Q. Liu, and Y. Li, "Fluidization characteristics of particles in a groove induced by horizontal air flow," Powder Technology, vol. 363, pp. 442-447, 2020.

[10] Z. Lin, X. Sun, T. Yu, Y. Zhang, Y. Li, and Z. Zhu, "Gas-solid two-phase flow and erosion calculation of gate valve based on the CFD-DEM model," Powder Technology, vol. 366, pp. 395-407, 2020.

[11] J.-Y. Tao, Z. Lin, C.-J. Ma et al., "An experimental and numerical study of regulating performance and flow loss in a V-port ball valve," Journal of Fluids Engineering, vol. 142, no. 2, 2020.

[12] X. Zheng, Z. Lin, and B. Y. Xu, “Thermal conductivity and sorption performance of nano-silver powder/FAPO-34 composite fin," Applied Thermal Engineering, vol. 160, Article ID 114055, 2019.

[13] G. Zhang, Y. F. Zhang, Q. Liu, Y. Li, and Z. Lin, "Experimental study on the two-phase flow of gas-particles through a model brake valve," Powder Technology, vol. 367, pp. 172-182, 2020.

[14] X. Sun, J. Liu, L. Ji et al., "A review on hydrodynamic cavitation disinfection: the current state of knowledge," Science of The Total Environment, vol. 737, Article ID 139606, 2020.

[15] X. Sun, X.-Q. Jia, J.- T Liu et al., "Investigation on the characteristics of an advanced rotational hydrodynamic cavitation reactor for water treatment," Separation and $\mathrm{Pu}$ rification Technology, vol. 251, Article ID 117252, 2020.

[16] Y. Zhang, J. Zhang, X. Lin, R. Wang, C. Zhang, and J. Zhao, "Experimental investigation into downstream field of a horizontal Axis tidal stream turbine supported by a mono pile," Applied Ocean Research, vol. 101, Article ID 102257, 2020.

[17] Y. Zhang, E. Fernandez-Rodriguez, J. Zheng et al., "A review on numerical development of tidal stream turbine performance and wake prediction," IEEE Access, vol. 8, pp. 79325-79337, 2020. 
[18] X. Li, T. Shen, P. Li, X. Guo, and Z. Zhu, "Extended compressible thermal cavitation model for the numerical simulation of cryogenic cavitating flow," International Journal of Hydrogen Energy, vol. 45, no. 16, pp. 10104-10118, 2020.

[19] T. Lin, Z. Zhu, X. Li, J. Li, and Y. Lin, "Theoretical, experimental, and numerical methods to predict the best efficiency point of centrifugal pump as turbine," Renewable Energy, vol. 168, no. 5, pp. 31-44, 2021.

[20] D. Ngomo, A. Chaudhuri, A. Chinnayya, and A. Hadjadj, "Numerical study of shock propagation and attenuation in narrow tubes including friction and heat losses," Computers \& Fluids, vol. 39, no. 9, pp. 1711-1721, 2010.

[21] G. Li, T. Ukai, and K. Kontis, "Characterization of a novel open-ended shock tube facility based on detonation transmission tubing," Aerospace Science and Technology, vol. 94, Article ID 105388, 2019.

[22] B. Henderson, J. Bridges, and M. Wernet, “An experimental study of the oscillatory flow structure of tone-producing supersonic impinging jets," Journal of Fluid Mechanics, vol. 542, no. -1, pp. 115-137, 2005.

[23] M. A. F. Kendall, "The delivery of particulate vaccines and drugs to human skin with a practical, hand-held shock tubebased system," Shock Waves, vol. 12, no. 1, pp. 23-30, 2002.

[24] M. A. F. Kendall, N. J. Quinlan, S. J. Thorpe, R. W. Ainsworth, and B.-J. Bellhouse, "Measurements of the gas and particle flow within a converging-diverging nozzle for high speed powdered vaccine and drug delivery," Experiments in Fluids, vol. 37, no. 1, pp. 128-136, 2004.

[25] Y. Liu, M.-A.-F. Kendall, N.-K. Truong, and B.-J. Bellhouse, "Numerical and Experimental Analysis of a High Speed Needle-free Powdered Vaccines Delivery Device," in Proceedings of the 20th AIAA Applied Aerodynamics Conference, St. Louis, Missouri, June 2002.

[26] X. Xiong, K. Gao, J. Zhang et al., "Interaction between shock wave and solid particles: establishing a model for the change of cloud's expansion rate," Powder Technology, vol. 381, pp. 632-641, 2021.

[27] R. Lupoi, M. Meyer, W. W. Wits, and S. Yin, "The role of particles flow characteristics in the performance of cold spray nozzles," CIRP Annals, vol. 69, no. 1, pp. 189-192, 2020.

[28] Z. Wang and H. Yan, "Unified gas-kinetic scheme for the monodisperse gas-particle flow and its application in the shock-driven multiphase instability," International Journal of Multiphase Flow, vol. 119, pp. 95-107, 2019.

[29] G. Zhang, I. Lee, T. Hashimoto, T. Setoguchi, and H. Kim, "Experimental study on gas-particle two-phase flows in a micro shock tube," Journal of Visualization, vol. 20, no. 1, pp. 17-29, 2017.

[30] G. Zhang, I. I. Lee, T. Hashimoto, T. Setoguchi, and H. D. Kim, "Experimental studies on shock wave and particle dynamics in a needle-free drug delivery device," Journal of Drug Delivery Science and Technology, vol. 41, pp. 390-400, 2017. 